\title{
Safety of the primary percutaneous coronary intervention strategy combining pre-hospital prasugrel, enoxaparin and in-hospital bivalirudin in acute ST-segment elevation myocardial infarction
}

\author{
Juho Viikilä**, Tuomo Nieminen², Ilkka Tierala² and Mika Laine ${ }^{2}$
}

\begin{abstract}
Backround: The optimal antithrombotic treatment during a primary percutaneous coronary intervention ( $\mathrm{PPCl}$ ) is not known. This single center registry study aims to assess the safety of a novel antithrombotic regimen combining enoxaparine and prasugrel at presentation, followed by bivalirudin at the catheterisation laboratory.

Methods: All consecutive patients who underwent a pPCI were collected prospectively. The primary endpoint was major bleeding within 30 days. The secondary endpoints were a composite of major adverse cardiovascular events (MACE) consisting of cardiovascular death, non-fatal myocardial infarction, non-fatal stroke, a new target vessel revascularisation and all-cause mortality at 30 days.

Results: Ninety-nine out of the total of 390 patients were treated according to the new regimen (protocol-treated group). The rest received other antithrombotic treatment (non-protocol-treated group). The protocol-treated group had a lower risk than the non-protocol-treated group according to the GRACE ischaemic (112 vs. 124, $p=0.002)$ and CRUSADE bleeding scores (21 vs. $28, p<0.0001)$. The incidences of bleeding were similar: severe GUSTO or TIMI bleeding occurred in $0 \%$ of the protocol-treated group and in 1.0 and $0.3 \%$, respectively, of the other group ( $p=0.311$ for GUSTO and $p=0.559$ for TIMI). The incidence of MACE in the groups was 6.1 and $10.7 \%$, respectively $(p=0.178)$. The respective incidences of all-cause mortality were 5.1 and $9.6 \%(p=0.158)$.
\end{abstract}

Conclusions: Administration of the novel antithrombotic regimen seems to be safe.

\section{Background}

Primary percutaneous coronary intervention (pPCI) is the preferred first-line treatment for acute ST- segment elevation myocardial infarction (STEMI) [1]. Antithrombotic treatment is an essential part of the pPCI procedure in enhancing the opening of the occluded coronary artery as well as preventing peri- and post-procedural thrombotic complications and late recurrent ischaemic events. Peri-procedural stroke, for example, has been recognised as an important life-limiting complication

\footnotetext{
*Correspondence: juho.viikila@phsotey.fi

'Department of Cardiology, Päijät-Häme Central Hospital, Keskussairaalankatu

7, 15850 Lahti, Finland

Full list of author information is available at the end of the article
}

[2]. The development of new antithrombotic agents has been rapid over the last decade. The application of clopidogrel and glycoprotein (GP) IIb/IIIa inhibitors in addition to heparin and aspirin has been shown to decrease early and late adverse cardiac events with a concomitant and undesired increase in bleeding events [3-6]. Bleeding has been recognised as a major determinant of cardiovascular death and adverse events in acute coronary syndrome (ACS) patients [7].

Prasugrel is a novel thrombocyte receptor P2Y12 inhibitor, which has a more rapid, efficacious and consistent antithombotic effect than clopidogrel $[8,9]$. In the TRITON TIMI-38 trial, prasugrel was more effective than clopidogrel in reducing adverse cardiac events in 
both acute non-ST-elevation myocardial infarction and STEMI patients [10]. However, the incidence of severe bleeding events, especially in elderly and low-weight patients as well as those with prior ischaemic cerebrovascular events was raised with prasugrel when compared to clopidogrel.

Bivalirudin is an intravenously administered direct, short-acting thrombin inhibitor [11]. In the HORIZONSAMI trial, it reduced severe bleeding events as well as early and late net adverse events in STEMI patients when compared to heparin plus GPIIb/IIIa-inhibitor, with an increase in the incidence of early stent thromboses [12]. In the EUROMAX study, bivalirudin started during transport for pPCI was similarily associated with a reduction in major bleeding events and an increase in early stent thrombosis compared to heparin or enoxaparin with optional GPIIb/IIIa inhibitor [13]. The recent HEAT-PPCI compared heparin and bivalirudin in a randomised settings; heparin reduced the incidence of major ischaemic events with equal safety profile [14]. Importantly, the other antithrombotic drugs used differed between the studies.

Enoxaparine is an alternative to unfractionated heparin. In the ATOLL trial, intravenous enoxaparine, in comparison to heparin, was associated with fewer ischaemic events with similar bleeding rates for acute STEMI patients undergoing pPCI [15].

No previous study has combined prehospital prasugrel and enoxaparine with bivalirudin. We hypothesized that using this combination of drugs might balance the risk of early stent thrombosis and bleeding events in pPCI patients. Thus, we report the results of our new antithrombotic pPCI regimen including aspirin, a low-dose enoxaparine i.v. bolus and prasugrel loading upon first medical contact (FMC) combined with a bivalirudin infusion initiated in the catheterisation laboratory.

\section{Methods}

\section{Study patients and data collection}

The present study took place in the Meilahti hospital, responsible of all PCIs for STEMI in the HelsinkiUusimaa Hospital District of 1.5 million inhabitants. According to the new local STEMI guidelines launched on 1 November 2010, all acute STEMI patients referred to pPCI should receive aspirin $250 \mathrm{mg}$, enoxaparine $30 \mathrm{mg}$ intravenously and prasugrel $60 \mathrm{mg}$ upon FMC. The bivalirudin infusion (a bolus of $0.75 \mathrm{mg} / \mathrm{kg}$ followed by an infusion of $1.75 \mathrm{mg} / \mathrm{kg} / \mathrm{h}$ ) should be started in the catheter laboratory. After PCI, aspirin $100 \mathrm{mg}$ daily should be continued indefinitely and prasugrel $10 \mathrm{mg}$ daily for 12 to 15 months. A lower prasugrel dosage of $5 \mathrm{mg}$ daily, or clopidogrel $75 \mathrm{mg}$ daily instead of prasugrel, is recommended for patients weighing under $60 \mathrm{~kg}$ or aged over 75 years. Those with a previous stroke or transient ischemic attack are recommended to continue with clopidogrel $75 \mathrm{mg}$ daily. When switching to clopidogrel, it is advised to load the new drug at 300-600 mg the day after prasugrel loading.

In order to assess the efficacy and safety of the new antithrombotic regimen, we examined all acute STEMI patients treated with $\mathrm{pPCI}$ in the Meilahti hospital between 1 January 2011 and 30 April 2012. The patients were prospectively collected into a local STEMI registry. All hospital files regarding the index STEMI hospitalisation and the following 30-day period were searched to receive detailed data on patients risk profile, treatments and clinical outcome. Mortality data was available for all patients from the National Population Register Centre. This was a registry study, which did not need an approval from the ethics committee. An informed consent was not needed; no contact to the patient was taken in this registry study and the data was anonymized and deidentified prior to analysis. The study protocol was approved by the Helsinki-Uusimaa Hospital District. The study was performed in accordance with the Declaration of Helsinki.

The inclusion criteria were acute STEMI treated with pPCI within the first 12 hours after symptom onset. The criteria for a STEMI diagnosis were acute chest pain (or equivalent) and 1) ST elevations of $\geq 2 \mathrm{~mm}$ ( $\geq 1.5 \mathrm{~mm}$ for women) in at least two of the leads V1-3, or 2) ST elevations of $\geq 1 \mathrm{~mm}$ in at least two other leads (V4-6, V8-9, V4R, I, aVL, II, III, aVF), or 3) a new left bundle branch block in ECG. Patients who received fibrinolysis were excluded from the study.

\section{Definitions of study endpoints}

The primary endpoint was major bleeding within 30 days. Bleedings were classified and reported according to GUSTO and TIMI criteria [16, 17]. The secondary endpoints were 1) a composite of major adverse cardiovascular events (MACE) consisting of cardiovascular death, non-fatal myocardial infarction, non-fatal stroke and a new target vessel revascularisation procedure, and 2) allcause mortality at 30 days. Myocardial infarction was defined according to current international guidelines [18]. Stroke was defined as any focal neurological deficit of ischaemic or haemorrhagic origin lasting for longer than 24 hours.

\section{Statistical analysis}

The follow-up of the acute STEMI patients who received the guideline-defined adjuvant treatment was analysed primarily without a control group. Secondarily, a comparison with pPCI patients who had received other adjuvant treatment was performed. Continuous variables are described using medians, means and standard deviations (SD). Categorical variables are described with absolute 
(number) and relative (percentage) frequency distribution. Statistical analyses across the groups were done with a chi-square test for categorical variables and ANOVA for continuous variables. A Cox regression survival analysis was performed for MACE using age, sex, access site, thrombectomy, antithrombotic treatment as well as GRACE and CRUSADE scores as covariates. IBM SPSS Statistics version 19.0 was used for all analyses.

\section{Results}

In total, 390 acute STEMI patients fulfilling the study criteria were identified within the study period. Ninetynine patients $(25 \%)$ received the complete guidelinedefined antithrombotic treatment with aspirin, a bolus of i.v. enoxaparine and a loading dose of prasugrel at presentation followed by a bivalirudin infusion at the catheter laboratory (protocol-treated group). The remaining 291 patients received some other combination of antithrombotic agents (non-protocol treated group).

The patients in the protocol-treated group were significantly younger and less likely to be on warfarin treatment at presentation than the patients in the non-protocol-treated group (Table 1). There was a trend towards a higher prevalence of diabetes and prior coronary artery bypass operations in the nonprotocol-treated group. Smoking was more common

Table 1 Baseline characteristics, cardiovascular risk factors, prior cardiovascular diseases, revascularisations and medications in the protocol and non-protocol treated groups

\begin{tabular}{|c|c|c|c|}
\hline & $\begin{array}{l}\text { Protocol } \\
n=99\end{array}$ & $\begin{array}{l}\text { Non-protocol } \\
n=291\end{array}$ & p \\
\hline Mean age, years, mean (SD) & $59.5(14)$ & $66.1(14)$ & $<0.001$ \\
\hline Age $>75$ years, $n(\%)$ & $11(11.1)$ & $97(33.3)$ & $<0.001$ \\
\hline Male sex, $n(\%)$ & $74(74.7)$ & $198(68.0)$ & 0.210 \\
\hline Diabetes, n (\%) & $13(13.1)$ & $64(22.0)$ & 0.056 \\
\hline Current smoker, $n(\%)$ & $50(50.5)$ & 95 (32.6) & 0.001 \\
\hline Hypertension, $n$ (\%) & $49(49.5)$ & $158(54.3)$ & 0.408 \\
\hline Dyslipidaemia, n (\%) & 39 (39.4) & $123(42.3)$ & 0.616 \\
\hline Renal dysfunction ${ }^{\mathrm{a}}, n(\%)$ & $3(3.0)$ & $11(3.8)$ & 0.729 \\
\hline Peripheral vascular disease, $n(\%)$ & $4(4.0)$ & $14(4.8)$ & 0.752 \\
\hline Previous myocardial infarction, $n$ (\%) & $9(9.1)$ & $32(11.0)$ & 0.593 \\
\hline Previous stroke, $n(\%)$ & $4(4.0)$ & $21(7.2)$ & 0.265 \\
\hline Previous CABG, $n(\%)$ & $1(1.0)$ & $15(5.2)$ & 0.073 \\
\hline Previous $\mathrm{PCl}, n(\%)$ & $10(10.1)$ & 39 (13.4) & 0.392 \\
\hline Aspirin, $n(\%)$ & $21(21.2)$ & $81(27.8)$ & 0.195 \\
\hline P2Y12-receptor inhibitor, n (\%) & $2(2.0)$ & $11(3.8)$ & 0.399 \\
\hline Warfarin, n (\%) & $2(2.0)$ & $37(12.7)$ & 0.002 \\
\hline
\end{tabular}

$C A B G$ coronary artery by-pass intervention, $\mathrm{PCl}$ percutaneous coronary intervention

a estimated glomerular filtration rate $<60 \mathrm{ml} / \mathrm{min} / 1.73 \mathrm{~m}^{2}$ in the protocol-treated group. Other cardiovascular risk factors or prior cardiovascular disease did not vary significantly across the groups (Table 1 ). At presentation, according to the GRACE and CRUSADE score calculations, the predicted risk of short- and long-term adverse cardiovascular events and inhospital bleeding events was significantly higher in the non-protocol-treated group (Table 2). The proportion of patients presenting with acute heart failure was also significantly higher in the non-protocol-treated group.

Sixteen percent of patients in the non-protocol-treated group had normal coronary arteries or only modest coronary artery lesions, whereas all patients in the protocoltreated group had significant coronary artery disease. Consequently, normal coronary artery flow before PCI was more frequent in the non-protocol-treated group (Table 2). However, the prevalence of left-main or treevessel disease did not vary significantly across the

Table 2 Clinical characteristics at presentation, coronary angiography and revascularisation procedures in the protocol and non-protocol treated groups

\begin{tabular}{|c|c|c|c|}
\hline & Protocol & Non-protocol & $p$-value \\
\hline & $n=99$ & $n=291$ & \\
\hline Anterior STEMI, $n(\%)$ & $43(43.4)$ & $138(47.4)$ & 0.492 \\
\hline GRACE score, mean (SD) & $112(31)$ & $124(36)$ & 0.002 \\
\hline CRUSADE score, mean (SD) & $21(13)$ & $28(16)$ & $<0.0001$ \\
\hline Killip class > I, n (\%) & $8(8.1)$ & $62(21.5)$ & 0.003 \\
\hline Killip class IV, n (\%) & $2(2.0)$ & $13(4.5)$ & 0.270 \\
\hline eGFR ml/min, mean (SD) & $103(40)$ & $90(41)$ & 0.009 \\
\hline Hemoglobin g/l, mean (SD) & $135(15)$ & $133(18)$ & 0.283 \\
\hline Weight kg, mean (SD) & $81(16)$ & $80(17)$ & 0.644 \\
\hline \multicolumn{4}{|l|}{ Angiography } \\
\hline Radialis access, $n(\%)$ & 33 (33.3) & 77 (26.6) & 0.196 \\
\hline $3 \mathrm{VD}, n(\%)$ & $14(14.1)$ & $56(19.2)$ & 0.253 \\
\hline LMD, $n(\%)$ & $2(2.0)$ & $16(5.5)$ & 0.154 \\
\hline TIMI flow grade $0-1, n(\%)$ & $68(70.1)$ & $119(57.2)$ & 0.031 \\
\hline TIMI flow grade $3, n(\%)$ & $15(15.5)$ & $58(27.9)$ & 0.018 \\
\hline $\begin{array}{l}\text { Normal findings or modest } \\
\text { coronary artery disease }\end{array}$ & $0(0)$ & $46(16.0)$ & $<0.0001$ \\
\hline $\mathrm{PCl}, n(\%)$ & $99(100)$ & $205(70.4)$ & $<0.0001$ \\
\hline Use of stents (of all PCI), $n$ (\%) & $91(91.9)$ & $187(91.2)$ & 0.838 \\
\hline Use of DES (of stents), $n$ (\%) & $13(14.3)$ & $24(12.8)$ & 0.738 \\
\hline Thrombectomy (of all PCI), n (\%) & $53(53.5)$ & $68(33.2)$ & 0.001 \\
\hline TIMI flow grade $0-1$ post PCI, $n(\%)$ & $0(0)$ & $8(3.8)$ & 0.053 \\
\hline TIMI flow grade 3 post PCI, $n(\%)$ & $89(91.8)$ & $182(85.8)$ & 0.143 \\
\hline CABG, $n(\%)$ & $2(2.0)$ & $17(5.8)$ & 0.127 \\
\hline
\end{tabular}

eGFR glomerular filtration rate estimated by Cockcroft-Gault formula, 3VD three vessel coronary artery disease, $L M D$ left main coronary artery disease, $L A D-P C I$ percutaneous coronary intervention to left anterior descending artery, DES drug-eluting stent, $C A B G$ coronary artery bypass graft surgery 
groups. A percutaneous coronary intervention was performed more often in the protocol-treated group. The use of bare metal or drug-eluting stents among the PCItreated patients did not vary significantly across the groups. However, thrombectomy was more common in the protocol-treated group. There were no significant differences in post-PCI coronary artery flow between the groups. The median total ischaemic time (delay from symptom onset to PCI) was $300 \mathrm{~min}$. and $264 \mathrm{~min}$. in the protocol-treated and non-protocol-treated groups, respectively ( $p=0.165$ between the groups).

The use of glycoprotein-inhibitors was uncommon in the protocol-treated group, whereas they were used in approximately one quarter of the patients in the nonprotocol-treated group (Table 3). Clopidogrel was used frequently in the non-protocol-treated group.

Bleeding events were rare in the overall population, and their occurrence did not differ between the groups (Table 4). None of the patients suffered intracranial or fatal bleedings during the study period. Major adverse cardiac events and deaths were observed more frequently in the non-protocol-treated group, but the difference across the groups was not statistically significant (Table 4). In the multivariable Cox analysis for MACE, high GRACE and CRUSADE scores were the only variables associated with worse prognosis (data not shown).

At discharge, prasugrel was used in 77 and $26 \%$ of the patients in the protocol-treated and non-protocoltreated groups, respectively, $(p<0.0001$ between the groups) and clopidogrel in 17 and $46 \%$ of the patients, respectively $(p<0.001)$. Statins were used in 94 and $82 \%(p=0.004)$, beta blockers in 89 and $78 \%(p=0.04)$ and angiotensin convertase inhibitors or angiotensin II receptor blockers in 81 and $65 \%$ of the patients $(p=0.003)$, respectively.

Table 3 Antithrombotic treatment in the protocol $(n=99)$ and non-protocol $(n=291)$ treated groups

\begin{tabular}{llll}
\hline & $\begin{array}{l}\text { Protocol, } \\
n(\%)\end{array}$ & $\begin{array}{l}\text { Non-protocol, } \\
n(\%)\end{array}$ & P-value \\
\hline First medical contact & $99(100)$ & $257(88.3)$ & $<0.0001$ \\
Aspirin & $99(100)$ & $139(47.8)$ & $<0.0001$ \\
Prasugrel loading dose & $1(1.0)$ & $116(39.9)$ & $<0.0001$ \\
Clopidogrel loading dose & $99(100)$ & $192(66.0)$ & $<0.0001$ \\
Enoxaparin bolus i.v. & $0(0)$ & $15(5.2)$ & 0.021 \\
Glycoprotein Ilb/IIla inhibitor & & & \\
Catheter laboratorio & $99(100)$ & $36(12.4)$ & $<0.0001$ \\
Bivalirudin & $6(6.1)$ & $79(27.1)$ & $<0.0001$ \\
Glycoprotein Ilb/IIla inhibitor & $9(9.1)$ & $56(19.2)$ & 0.019 \\
Enoxaparin i.v. & $0(0)$ & $7(2.4)$ & 0.119 \\
Unfractionated heparin & & &
\end{tabular}

Table 4 Major adverse cardiac events, mortality and bleeding events at 30 days in the protocol $(n=99)$ and non-protocol $(n=291)$ treated groups

\begin{tabular}{llll}
\hline & $\begin{array}{l}\text { Protocol } \\
n(\%)\end{array}$ & $\begin{array}{l}\text { Non-protocol } \\
n(\%)\end{array}$ & $p$-value \\
\hline MACE & $6(6.1)$ & $31(10.7)$ & 0.178 \\
Cardiovascular death & $4(4.0)$ & $26(8.9)$ & 0.114 \\
Non-fatal myocardial infarction & $0(0)$ & $1(0.3)$ & 0.559 \\
Non-fatal stroke & $1(1.0)$ & $4(1.4)$ & 0.781 \\
Target vessel revascularisation & $1(1.0)$ & $3(1.0)$ & 0.986 \\
Death from any cause & $5(5.1)$ & $28(9.6)$ & 0.158 \\
Severe GUSTO bleeding & $0(0)$ & $3(1.0)$ & 0.311 \\
$\quad$ Mild or minor GUSTO bleeding & $3(3.0)$ & $6(2.1)$ & 0.579 \\
Major TIMI bleeding & $0(0)$ & $1(0.3)$ & 0.559 \\
Minor or minimal TIMI bleeding & $2(2.0)$ & $4(1.4)$ & 0.652 \\
\hline
\end{tabular}

\section{Discussion}

\section{Bleeding events}

In this paper, we report the results on STEMI patients treated with a novel antithrombotic regimen combining aspirin, an i.v. enoxaparine bolus and a prasugrel loading dose at presentation, followed by bivalirudin infusion at the catheterisation laboratory. To the best of our knowledge, no previous study has used the same adjuvant therapy. Our principal finding is that the administration of the new regimen is safe. None of the protocol-treated patients suffered a severe bleeding event during the 30-day follow-up. The incidence of mild to moderate bleeding events was only $3 \%$ in that group. This outcome was favourable compared to the CRUSADE-score-based estimation of $5.5 \%$ inhospital incidence of bleeding events in the protocol treated group. On the other hand, the incidence of severe bleeding events was low also in the non-protocoltreated group as well, and there were no significant differences in bleeding events between the groups. Therefore, our findings support the concept that bivalirudin can be combined to low-dose low-molecular heparin without increasing bleeding complications.

The safety of bivalirudin compared to heparin plus GP IIb/IIIa-inhibitors in pPCI-patients is supported by two major trials: HORIZONS-AMI and EUROMAX [12, 13]. The incidence of net adverse and severe bleeding events was reduced with bivalirudin in both trials. In HORIZONS-AMI, bivalirudin was compared to heparin plus GP-inhibitors with almost all patients having a clopidogrel loading at the time of admission. Heparin was given in $68 \%$ in the bivalirudin group. In the Euromax study, $51 \%$ of the patients had a clopidogrel loading as soon as possible and the rest received either prasugrel (30\%) or ticagrelor (19\%). Heparin was used in only $2.2 \%$ in the bivalirudin group. 


\section{Adverse events}

None of the protocol-treated patients suffered from stent thrombosis or recurrent myocardial infarction. The non-protocol group presented one stent thrombosis and one myocardial infarction within a month. The incidence of MACE and all-cause death in the protocol-treated group was 6.1 and 5.1 percent, respectively. These numbers are comparable to MACE and mortality figures recently published based on the French and Swedish STEMI registries $[19,20]$. From this perspective, the new anti-thrombotic regimen also seems to be efficient.

The non-protocol group presented with a higher risk profile (older age, warfarin usage, higher GRACE and CRUSADE scores, higher Killip class at presentation, worse renal function) than those treated with the new protocol. However, the number of endpoints did not differ between the treatment groups, which is probably due to the low number of adverse events. The Cox regression analysis presented the GRACE and CRUSADE scores - which integrate patient-related information from several angles - as the only variables predicting poor prognosis.

The rate of early stent thromboses was increased with bivalirudin in both the HORIZONS-AMI and EUROMAX trials. This may be partly due to an inconsistent action of clopidogrel used widely in both trials. Importantly, in HORIZONS-AMI, those patients who had received the heparin bolus prior to randomisation had a lower risk of stent thrombosis than those without prerandomisation heparin [21]. In EUROMAX, only $2 \%$ of the bivalirudin treated patients were protected by heparin. However, in our study, the usage of prasugrel and enoxaparine with bivalirudin might have protected patients against stent thromboses.

The recent HEAT-PPCI study randomised STEMI patients to receive either bivalirudin or heparin infusion, with provisional GP IIb/IIIa inhibitors used in only 13$15 \%$ of the patients in each group [14]. Only $11 \%$ received clopidogrel, as prasugrel (27 \%) and ticagrelor (62\%) were clearly more common. The rate of major adverse events during 28 day follow-up was significantly higher with bivalirudin mainly due to increased rate of stent thromboses. The bleeding rates did not differ across the groups. This might be due to low usage of GP IIb/IIIa inhibitors also in the heparin arm. Of note, the patients in the bivalirudin group were not protected with either heparin or enoxaparine.

Our hypothesis was that combining pre-hospital enoxaparine and prasugrel with bivalirudin during the pPCI procedure might balance the risk of bleeding and early stent thromboses. The observed low incidence of bleeding events and the lack of stent thromboses and recurrent myocardial infarctions in the protocol-treated patients support the hypothesis. We replaced heparin, which was widely used in the bivalirudin arm in the HORIZONS-AMI trial, by a low dose of enoxaparine at FMC without any evident rise in bleeding events.

\section{Adoption of the new protocol}

The rate of adoption of the new local guidelines in daily practice was clearly lower than we expected. The deviations were mostly due to the omission of prasugrel and, particularly, bivalirudin. Firstly, only half of the patients in the non-protocol-treated group received prasugrel at FMC. This might reflect the fact that we started the survey period relatively soon, two months after the guideline implementation. Patients were referred to our pPCI centre from a relatively large area with different emergency medical system organisations, a setting that may have compromised the early guideline implementation process. Secondly, the utilisation of bivalirudin in the catheter laboratory was low in the non-protocol-treated group. Operators seemed to prefer to see the angiographic result before deciding whether to use bivalirudin. This policy is reflected as a higher incidence of normal angiographic findings in the non-protocoltreated groups.

\section{Limitations}

We observed unexpectedly low bleeding rates in both treatment groups. Despite the differences in GRACE and CRUSADE scores the rate of adverse events did not differ across the groups. These findings might be related to the relatively small sample size and the non-randomised trial design, which are limitations to our study. Another limitation is the low penetration of the guidelines in daily practice, leaving open questions on the usability of the new regimen in unselected patient populations.

\section{Conclusions}

The present study offers preliminary findings on a new antithrombotic regimen combining low-dose enoxaparine, prasugrel and bivalirudin in STEMI patients. The data are promising and suggest that the regimen is both safe and efficient, but the results need to be confirmed in randomised studies.

\section{Abbreviations \\ ACS, acute coronary syndrome; CABG, coronary artery by-pass graft surgery; FMC, first medical contact; Gp-inhibitor, glycoprotein receptor inhibitor; MACE, major adverse cardiovascular event; $\mathrm{PCl}$, percutaneous coronary intervention; STEMI, ST-segment elevation myocardial infarction}

Acknowledgements

We thank Eeva Parviainen who performed English language revision.

Funding

None.

Availability of data and materials

Data will be shared on request. 


\section{Authors' contributions}

$\mathrm{JV}$ drafted the manuscript, performed the statistical analysis and participated in the design and conception of the study, TN made main contribution to conception and design of the study, performed data collection and helped to draft the manuscript and make the statistical analysis. IT participated in the design and coordination of the study and participated in the data collection. ML participated in the design and coordination of the study and helped to draft the manuscript. All authors have read and accepted the manuscript.

\section{Competing interests}

The authors declare that they have no competing interests.

\section{Consent for publication}

Not applicable.

\section{Ethics approval and consent to participate}

This was a registry study, which did not need an approval from the ethics committee. An informed consent was not needed; no contact to the patient was taken in this registry study and the data was anonymized and de-identified prior to analysis. The study protocol was approved by the Helsinki-Uusimaa Hospital District. The study was performed in accordance with the Declaration of Helsinki.

\section{Author details}

${ }^{1}$ Department of Cardiology, Päijät-Häme Central Hospital, Keskussairaalankatu 7, 15850 Lahti, Finland. ${ }^{2}$ Department of Cardiology, Helsinki University Central Hospital, Helsinki, Finland.

Received: 31 January 2016 Accepted: 21 July 2016

Published online: 30 July 2016

\section{References}

1. The Task Force on the management of ST-segment elevation acute myocardial infarction of the European Society of Cardiology (ESC) Authors/ Task Force Members, Steg G, James SK, Atar D, Badano LP, BlöstromLundqvist C, Bogerm, et al. ESC guidelines for the management of acute myocardial infarction in patients presenting with ST-segment elevation. Eur Heart J. 2012;33:2569-19.

2. D'Ascenzo F, Barbero U, Moretti C, Plamerini T, Della Riva D, Mariani A, et al. Percutaneous coronary intervention versus coronary artery bypass graft for stable angina: meta-regression of randomized trials. Contemp Clin Trials. 2014;28:51-8.

3. Yusuf S, Zhao F, Mehta SR, Chrolavicius S, Tognoni G, Fox KK. Clopidogrel in unstable angina to prevent recurrent events trial investigators. Effects of clopidogrel in addition to aspirin in patients with acute coronary syndromes without ST-segment elevation. N Engl J Med. 2001;345:494-502.

4. Chen ZM, Jiang LX, Chen YP, Xie JX, Pan HC, Peto R, COMMIT (ClOpidogrel and Metoprolol in Myocardial Infarction Trial) collaborative group, et al. Addition of clopidogrel to aspirin in 45,852 patients with acute myocardial infarction: randomised placebo-controlled trial. Lancet. 2005;366:1607-21.

5. Mehta SR, Tanguay JF, Eikelboom JW, Jolly SS, Joyner CD, Granger CB, et al. Double-dose versus standard-dose clopidogrel and high-dose versus lowdose aspirin in individuals undergoing percutaneous coronary intervention for acute coronary syndromes (CURRENT-OASIS 7): a randomised factorial trial. Lancet. 2010;376:1233-43.

6. De Luca G, Navarese E, Marino P. Risk profile and benefits from Gp Ilb-Illa inhibitors among patients with ST-segment elevation myocardial infarction treated with primary angioplasty: a meta-regression analysis of randomized trials. Eur Heart J. 2009;30:2705-13.

7. Eikelboom JW, Mehta SR, Anand SS, Xie C, Fox KA, Yusuf S. Adverse impact of bleeding on prognosis in patients with acute coronary syndromes. Circulation. 2006:114:774-82.

8. Niitsu Y, Jakubowski JA, Sugidachi A, Asai F. Pharmacology of CS-747 (prasugrel, LY640315), a novel, potent antiplatelet agent with in vivo P2Y12 receptor antagonist activity. Semin Thromb Hemost. 2005;31:184-94.

9. Jernberg T, Payne CD, Winters KJ, Darstein C, Brandt JT, Jakubowski JA. Prasugrel achieves greater inhibition of platelet aggregation and a lower rate of non-responders compared with clopidogrel in aspirin-treated patients with stable coronary artery disease. Eur Heart J. 2006;27:1166-73.
10. Montalescot G, Wiviott SD, Braunwald E, Murphy SA, Gibson CM, McCABE $\mathrm{CH}$, et al. Prasugrel compared with clopidogrel in patients undergoing percutaneous coronary intervention for ST-elevation myocardial infarction (TRITON TIMI 38): double-blind, randomised controlled trial. Lancet. 2009;373:723-31.

11. Warkentin TE, Greinacher A, Koster A. Bivalirudin. Thromb Haemost. 2008;99:830-9.

12. Stone GW, Witzenbichler B, Guagliumi G, Peruga JZ, Brodie BR, Dudek D, et al. Bivalirudin during primary $\mathrm{PCl}$ in acute myocardial infarction. N Engl J Med. 2008;358:2218-30.

13. Steg PG, van't Hof A, Hamm CW, Clemmensen P, Lapostelle F, Coste $P$, et al. Bivalirudin started during emergency transport for primary PCI. N Engl J Med. 2013;369:2207-17.

14. Shahzad A, Kemp I, Mars C, Wilson K, Roome C, Cooper R, et al. Unfractionated heparin versus bivalirudin in primary percutaneous coronary intervention (HEAT-PPCI); an open-label, single centre, randomised controlled trial. Lancet. 2014;384:1849-58.

15. Montalescot G, Zeymer U, Silvain J, Boulanger B, Cohen M, Goldstein P, ATOLL Investigators et al. Intravenous enoxaparin or unfractionated heparin in primary percutaneous coronary intervention for ST-elevation myocardial infarction: the international randomised open-label ATOLL trial. Lancet. 2011:378:693-703.

16. The GUSTO investigators. An international randomized trial comparing four thrombolytic strategies for acute myocardial infarction. N Engl J Med. 1993;329:673-82.

17. Chesebro JH, Knatterud G, Roberts R, Borer J, Cohen LS, Dalen J, et al. Thrombolysis in Myocardial Infarction (TIMI) Trial, Phase I: a comparison between intravenous tissue plasminogen activator and intravenous streptokinase. Clinical findings through hospital discharge. Circulation. 1987;76:142-54

18. Thygesen K, Alpert JS, Jaffe AS, Simoons ML, Chaitman BR, White HD, Joint ESC/ACCF/AHA/WHF Task Force for the Universal Definition of Myocardial, et al. Third universal definition of myocardial infarction. Eur Heart J. 2012;33:2551-67.

19. Hanssen M, Cottin $Y$, Khalife $K$, Hammer L, Goldstein $P$, Puymirat $P$, for the FAST-MI 2010 investigators et al. French registry on acute ST-elevation and non ST-elevation myocardial infarction 2010. FAST-MI 2010. Heart. 2012;98:699-705.

20. Stenestrand U, Lindback J, Wallentin L, RIKS-HIA Registry. Long-term outcome of primary percutaneous coronary intervention vs prehospital and in-hospital thrombolysis for patients with ST-elevation myocardial infarction. JAMA. 2006;296:1749-56

21. Dangas GD, Caixeta A, Mehran R, Parise H, Lansky AJ, Cristea E, et al. Harmonizing Outcomes With Revascularization and Stents in Acute Myocardial Infarction (HORIZONS-AMI) trial investigators. Frequency and predictors of stent thrombosis after percutaneous coronary intervention in acute myocardial infarction. Circulation. 2011;123:1745-56.

\section{Submit your next manuscript to BioMed Central and we will help you at every step:}

- We accept pre-submission inquiries

- Our selector tool helps you to find the most relevant journal

- We provide round the clock customer support

- Convenient online submission

- Thorough peer review

- Inclusion in PubMed and all major indexing services

- Maximum visibility for your research

Submit your manuscript at www.biomedcentral.com/submit
) Biomed Central 